\title{
Variational principles of second, first and intermediate kinds for non-holonomic mechanics*
}

\author{
ZHU Ruzeng (朱如曾) \\ (Laboratory for Nonlinear Mechanics of Continuous Media, Institute of Mechanics, Chinese \\ Academy of Sciences, Beijing 100080. China) \\ Received Deceniber 5, 1998
}

\begin{abstract}
The differential variational principles of second kind for non-holonomic mechanics are given, from which a number of integral variational principles of second kind are set up. From the latter, the general relation of $\delta q^{\prime}-\delta q$ and the general form of integral variational principles of the first kind and intermediate kinds gie deriver. Thus not only all previous relations of $\delta q^{\prime}-\delta q$ and integral variational principles are unified hi: $\varepsilon^{\prime} s c$ the existante of the variatisnal principles of intermediate kinds are pointed out.
\end{abstract}

Keywords: non-holonomi: nerhasies, vaviationai princi-jic.

For the non-soionomic mechanics of freedom degree $n$, in state space, all the generalized coordinates $q=\left(q_{1}, \cdots, q_{n}\right)$ and generalized velocities $q^{\prime}=\left(q_{1}^{\prime}, \cdots, q_{n}^{\prime}\right)$ should be looked as basic variables and so the motion equations for $q_{s}$

$$
\dot{q}_{s}=q_{s}^{\prime} \quad(s=1, \cdots, n)
$$

should be put on a bar with the motion equations for $q_{s}^{\prime}$, i.e. the form of Newton's second law of motion in state space. Here the time differential of $q_{s}$ has been expressed by $\dot{q}_{s}$ for short. All the previous variational principles for non-holonomic mechanics ${ }^{[1-4]}$ can give the motions for $q^{\prime}$ only if those of $q$ (eq. (1)) are accepted beforehand, because only the generalized coordinates are permitted to get independent variation while the generalized velocities are not permitted so. In the present paper, the variational principles from which both the motion equations of $q$ and those of $q^{\prime}$ can be derived together will be set up for non-holonomic mechanics. The variational principles given in the present paper will be called variational principles of second kind because both the $\delta q^{\prime}$ and $\delta q$ are independent, while the previous variational principles for non-holonomic systems are called the variational principles of the first kind. Besides, in non-holonomic mechanics, many different relations of $\delta q^{\prime}-\delta q$ and different integral variational principles of the first kind were suggested and even many arguments appeared $^{[1-6]}$. Can all those relations and principles be unified? It will be shown in the present paper that our variational principles of the second kind for non-holonomic mechanics can lead to the general relation of $\delta^{\prime} q-\delta q$ and the general form of integral variational principles of the first kind and intermediate kinds for holonomic and non-holonomic mechanics. Thus not only all previous relations of $\delta^{\prime} q-\delta q$ and integral variational principles are unified with the result of canceling any possibility of arguments, but also the existance of the variational principles of intermediate kinds are pointed out.

* Project supported by the National Natural Science Foundation of China (Grant No. 19272064). 


\section{The differential variational principles of the second kind}

Consider a mechanical system composed of $N$ mass points subject to $m$ independent, ideal and holonomic constraints

$$
W_{a}(r, t)=0 \quad(\alpha=1, \cdots, m<3 N)
$$

and $g$ independent, non-holonomic, ideal (in Appell-Chetaev's sense ${ }^{[1-3]}$ ) constraints of first degree

$$
G_{\beta}\left(\boldsymbol{r}, \boldsymbol{r}^{\prime}, t\right)=0 \quad(\beta=1, \cdots, g<3 N-m),
$$

where $\boldsymbol{r}=\left(\boldsymbol{r}_{1}, \cdots, \boldsymbol{r}_{N}\right), \boldsymbol{r}^{\prime}=\left(\boldsymbol{r}_{1}^{\prime}, \cdots, \boldsymbol{r}_{N}^{\prime}\right)$, with $\boldsymbol{r}_{i}$ and $\boldsymbol{r}_{i}^{\prime}$ being the position vector and velocity vector of $i$ th mass point respectively. The forces of constraint $\boldsymbol{R}_{i}$ of ideal constraints possess the property: for vector elements $\delta \boldsymbol{r}\left(\delta \boldsymbol{r}_{1}, \cdots, \delta \boldsymbol{r}_{N}\right)$ satisfying

$$
\sum_{i=1}^{N} \frac{\partial W_{\alpha}}{\partial \boldsymbol{r}_{i}} \cdot \delta \boldsymbol{r}_{i}=0 \quad(\alpha=1, \cdots, m)
$$

and Appell-Chetaev's conditions ${ }^{[1-3]}$

$$
\sum_{i=1}^{N} \frac{\partial}{\partial \boldsymbol{r}_{i}^{\prime}} \boldsymbol{g}_{\beta}\left(\boldsymbol{r}, \boldsymbol{r}^{\prime}, t\right) \cdot \delta \boldsymbol{r}_{i}=0 \quad(\beta=1,2, \cdots, \boldsymbol{g}),
$$

we have

$$
\sum_{i=1}^{N} \boldsymbol{R}_{i} \cdot \delta \boldsymbol{r}_{i}=0
$$

This is equivalent to that the forces of constraint $\boldsymbol{R}_{i}$ exerted upon $i$ th mass point can be expressed as ${ }^{[3]}$

$$
\boldsymbol{R}_{i}=\sum_{a=1}^{m} \mu_{\alpha}(t) \frac{\partial W_{\alpha}}{\partial \boldsymbol{r}_{i}}+\sum_{\beta=1}^{g} \zeta_{\beta}(t) \frac{\partial}{\partial \boldsymbol{r}_{i}^{\prime}} G_{\beta}\left(\boldsymbol{r}, \boldsymbol{r}^{\prime}, t\right) \quad(i=1, \cdots, N),
$$

where the coefficients $\mu_{\alpha}$ and $\zeta_{\beta}$ depend on temporary dynamical states. In terms of state variables, Newton's second law of motion can be written as

$$
\begin{gathered}
\boldsymbol{F}_{i}+\boldsymbol{R}_{i}-m_{\imath} \frac{\mathrm{d}}{\mathrm{d} t} \boldsymbol{r}^{\prime}=0 \quad(i=1, \cdots, N), \\
\dot{\boldsymbol{r}}_{i}-\boldsymbol{r}_{i}^{\prime}=0 \quad(i=1, \cdots, N),
\end{gathered}
$$

where $m_{i}$ and $\boldsymbol{F}_{i}$ are the mass of $i$ th mass point and the active force exerted upon it respectively.

Theorem 1 (Newtonian representation of generalized $D$ ' Alembert-Lagrange's principle (differential variational principles of second kind)). The sufficiently smooth functions $(\boldsymbol{r}(\boldsymbol{t})$, $\left.\boldsymbol{r}^{\prime}(t)\right)$ defined on time interval $\left[t_{0}, t_{1}\right]$ is an actual motion of constrained Newtonian system represented by eqs. (2), (3) and (7)-(9), if and only if it satisfies eqs. (2), (3) and

$$
\sum_{i=1}^{N}\left(\boldsymbol{F}_{\boldsymbol{t}}-m_{i} \frac{\mathrm{d}}{\mathrm{d} t} \boldsymbol{r}_{i}^{\prime}\right) \cdot \delta \boldsymbol{r}_{i}+\sum_{i=1}^{N} m_{i}\left(\dot{\boldsymbol{r}}_{i}-\boldsymbol{r}_{i}^{\prime}\right) \cdot \delta \boldsymbol{r}_{i}^{\prime}=0
$$

for every moment of time $t \in\left[t_{0}, t_{1}\right]$, all vector elements $\delta r^{\prime}\left(\delta r_{1}^{\prime}, \cdots, \delta r_{N}^{\prime}\right)$ and all vector elements $\delta r$ satisfying conditions (4) and (5).

Proof. We first prove the necessity of the conditions. Suppose that $\left(r(t), r^{\prime}(t)\right)$ is an actual motion of constrained Newtonian system represented by eqs. (2), (3), (7)-(9) on time interval $\left[t_{0}, t_{1}\right]$. Then it satisfies conditions (2) and (3). For any vector elements $\delta \boldsymbol{r}^{\prime}$ and any vector elements $\delta \boldsymbol{r}$ satisfying conditions (4) and (5), we have eq. (6) from eq. (7). Eqs. (6), (8) and (9) give (10). This completes the proof of the necessity of the conditions.

Now we prove the sufficiency of the conditions. The conditions of the theorem give (2) and (3). 
Since $\boldsymbol{r}$ in (10) satisfies (4) and (5), we can use Lagrange's multiplier method with undetermined multipliers $\mu_{\alpha}$ and $\zeta_{\beta}$ to obtain (8) and (9). The sufficiency of the conditions has been proven.

Now we are going to express this principle in terms of generalized state coordinates $\left(q, q^{\prime}\right)$. The number of the generalized configuration coordinates $q_{s}$ on the intersection of hypersurfaces (2) is $n=$ $3 N-m$, i. e. whenever $\boldsymbol{r}$ satisfies eq. (2), we have generalized configuration coordinates $q_{i}=$ $q_{i}(\boldsymbol{r}, t)(i=1, \cdots, n)$. For the sake of convenience, for every $t \in\left(t_{0}, t\right)$ we extend smoothly and invertiblly these $n$ generalized configuration coordinates on the intersection of hypersurfaces (2) to an open field $\Sigma(t)$ in state space $\left(r, r^{\prime}\right)$ containing the intersection themselves, and then in $\Sigma(t)$ we define $m$ auxiliary configuration coordinates $q_{n+\alpha}=W_{\alpha}\left(r_{1}, \cdots, r_{N}, t\right)(\alpha=1, \cdots, m)$. The generalized-velocities coordinates $q^{\prime}\left(q_{1}^{\prime}, \cdots, q_{3 N}^{\prime}\right)$ are defined as

$$
q_{s}^{\prime}=\frac{\partial q_{s}}{\partial t}+\sum_{i=1}^{N} \frac{\partial q_{s}}{\partial \boldsymbol{r}_{\imath}} \cdot r_{i}^{\prime} \quad(s=1, \cdots, 3 N) .
$$

The inverse transformation is

$$
\boldsymbol{r}_{i}=\boldsymbol{r}_{i}(q, t), \boldsymbol{r}_{i}^{\prime}=\frac{\partial \boldsymbol{r}_{i}}{\partial t}+\sum_{i=1}^{3 N} \frac{\partial}{\partial q_{i}} \boldsymbol{r}_{i}^{\prime} \quad(i=1, \cdots, N)
$$

From eqs. (10)-(12), we sail easily prove the new relations

$$
\frac{\partial \boldsymbol{r}_{i}^{\prime}}{\partial q_{i}^{\prime}}=\frac{\partial \boldsymbol{r}_{i}}{\partial \eta_{s}}, \frac{d}{\mathrm{a} t} \frac{\partial-}{\partial q_{s}}=\frac{\partial \boldsymbol{r}^{\prime}}{\partial q_{s}}+\sum_{k=1}^{\infty}\left(\dot{q}_{k}-q_{k}^{\prime}\right) \frac{\partial^{2} \boldsymbol{r}_{i}}{\partial q_{s} \partial q_{k}}\left(\begin{array}{l}
i=1, \cdots, N \\
s=1, \cdots, n
\end{array}\right)
$$

corresponding to Lagrange relations and the new central equation

$$
\sum_{s=1}^{n}\left(-\frac{\mathrm{d}}{\mathrm{d} t} \frac{\partial T}{\partial q_{s}^{\prime}}+\frac{\partial T}{\partial q_{s}}+Q_{s}\right) \delta q_{s}+\sum_{s=1}^{n}\left(\dot{q}_{s}-q_{s}^{\prime}\right) \delta \frac{\partial T}{\partial q_{s}^{\prime}}=0
$$

where the definitions of kinetic energy $T$ and generalized force $Q_{s}\left(T=\sum_{i=1}^{N} \frac{1}{2} m_{i} \boldsymbol{r}_{i}^{\prime 2}\right.$ and $Q_{s}=\sum_{i=1}^{N} \boldsymbol{F}_{i}$ - $\left.\frac{\partial \boldsymbol{r}_{i}}{\partial q_{s}}\right)$ are used. Due to condition (2), $s$ and $k$ in (13) and (14) only take 1 to $n$ but not 1 to $3 N$ (From now on, $q$ and $q^{\prime}$ have only $n$ components). Eq. (14) becomes

$$
\sum_{s=1}^{n}\left(-\frac{\mathrm{d}}{\mathrm{d} t} \frac{\partial}{\partial q_{s}^{\prime}} L+\frac{\partial}{\partial q_{s}} L+Q_{s}^{\prime}\right) \delta q_{s}+\sum_{s=1}^{n}\left(\dot{q}_{s}-q_{s}^{\prime}\right) \delta \frac{\partial L}{\partial q_{s}^{\prime}}=0,
$$

where $L$ is Lagrangian $L=T-V$ containing the contributions of all conservative active forces and $Q^{\prime}$ s $(s=1, \cdots, n)$ are possible non-conservative forces. Based on these results with further trivial derivation, we obtain the following statement:

Theorem 2 (Lagrange representation of generalized D' Alembert-Lagrange's principle). The sufficiently smooth function $\left(q(t), q^{\prime}(t)\right)$ defined time interval $\left[t_{0}, t_{1}\right]$ is an actual motion of Lagrange system with function $L$ and constraint conditions

$$
f_{\beta}\left(q, q^{\prime}, t\right)=G_{\beta}\left(\boldsymbol{r}(q, t), \boldsymbol{r}^{\prime}\left(q, q^{\prime}, t\right), t\right)=0 \quad(\beta=1, \cdots, g)
$$

( $i$. e. conditions (2) and (3)), if and only if it satisfies (16) and (15) for every moment of time $t \in$ $\left[t_{0}, t_{1}\right]$, all $\delta q^{\prime}$ and all $\delta q$ satisfying conditions

$$
\sum_{s=1}^{n} \frac{\partial f_{\beta}}{\partial q_{s}^{\prime}} \delta q_{s}=0 \quad(\beta=1, \cdots, g)
$$

\section{Integral variational principles of second kind}

By using the obvious relation $\delta \dot{q}_{s}=\mathrm{d} / \mathrm{d} t \delta q_{s}$, eq. (14) can be written as 


$$
-\frac{\mathrm{d}}{\mathrm{d} t}\left(\sum_{s=1}^{n} \frac{\partial T}{\partial q_{s}^{\prime}} \delta q_{s}\right)+\delta T+\delta \sum_{s=1}^{n}\left(\dot{q}_{s}-q_{s}^{\prime}\right) \frac{\partial T}{\partial q_{s}^{\prime}}+\sum_{s=1}^{n} Q_{s}^{\prime} \delta q_{s}=0
$$

Now let us extend $q(t)$ and $q^{\prime}(t)$ to $t_{0}$ and $t_{1}$ continuously. By using the fundamental theorem for variational method ${ }^{[7]}$, we can easily prove that (18) for time interval $\left(t_{0}, t_{1}\right)$ is equivalent to

$$
\int_{t_{0}}^{t_{1}}\left\{\delta\left[L+\sum_{s=1}^{n}\left(\dot{q}_{s}-q_{s}^{\prime}\right) \frac{\partial L}{\partial q_{s}^{\prime}}\right]+\sum_{s=1}^{n} Q_{s}^{\prime} \delta q_{s}\right\} \mathrm{d} t-\left.\sum_{s=1}^{n} \frac{\partial L}{\partial q_{s}^{\prime}} \delta q_{s}\right|_{t_{0}} ^{t_{1}}=0,
$$

which is called "partial variational equation" because of the restrictions (17) on $\delta q$. Thus we have

Theorem 3 (Lagrangian representation of integral variational principle of second kind with free end-points). The sufficiently smooth function $\left(q(t), q^{\prime}(t)\right)$ defined on time interval $\left[t_{0}\right.$, $\left.t_{1}\right]$ is an actual motion of Lagrange system with constraint conditions (16) (i.e. conditions (2) and (3)), if and only if it satisfies eq. (16) for every moment of time $t \in\left[t_{0}, t_{1}\right]$ and the partial variational equation (19) with all $\delta q^{\prime}$ and all $\delta q$ satisfying conditions satisfy condition (17).

Under the end-point conditions

$$
\delta q_{s}\left(t_{0}\right)=\delta q_{s}\left(t_{1}\right)=0(s=1, \cdots, n)
$$

or under condition $(20)$ and

$$
\varepsilon_{i s}^{\prime}\left(i_{0}\right):=\delta q_{s}^{\prime}\left(t_{1}\right):=0(\varepsilon=1, \cdots, n),
$$

generalized D'Alember-iajrange's principle remains tenable in the open interval $\left(t_{0}, t_{1}\right)$, and so the integral variatiorifl pinciple aiso remains tenable; only the forms of eq. (19) become

$$
\int_{t_{0}}^{t_{1}}\left\{\delta\left[L+\sum_{s=1}^{n}\left(\dot{q}_{s}-q_{s}^{\prime}\right) \frac{\partial L}{\partial q_{s}^{\prime}}\right]+\sum_{s=1}^{n} Q_{s}^{\prime} \delta q_{s}\right\} \mathrm{d} t=0 .
$$

Thus we have the following statement:

Theorem 4 (Lagrange representation of integral variational principle of second kind with half-fixed (or fixed, end points)). The sufficiently smooth function $\left(q(t), q^{\prime}(t)\right)$ defined on time interval $\left[t_{0}, t_{1}\right]$ is an actual motion of Lagrange system with constraint condition (16) (i.e. conditions (2) and (3)), if and only if it satisfies eq. (16) for every moment of time $t \in\left[t_{0}, t_{1}\right]$ and the partial variational equation (22) with all $\delta q^{\prime}$ ( or satisfying condition (21)) and all $\delta q$ satisfying conditions (17) and (20).

By using Lagrange's multiplier $\left(\lambda_{\beta}\right)$ method and considering the non-singularity of Hertz matrix $\frac{\partial^{2} T}{\partial q^{\prime} \partial q^{\prime}}$, the above integral variational principle of the second kind gives the complete motion equations in state space: eqs. (16) and

$$
\begin{gathered}
-\frac{\mathrm{d}}{\mathrm{d} t} \frac{\partial T}{\partial q_{s}^{\prime}}+\frac{\partial T}{\partial q_{s}}+Q_{s}+\sum_{\beta=1}^{g} \lambda_{\beta} \frac{\partial f_{\beta}}{\partial q_{s}^{\prime}}=0(s=1, \cdots, n), \\
\dot{q}_{s}=q_{s}^{\prime} \quad(s=1, \cdots, n) .
\end{gathered}
$$

Substituting (24) into (23) gives well-known Routh equations ${ }^{[1-3]}$, which also shows the correctness of our variational principles of the second kind.

\section{Hamilton representation of the variational principle of the second kind}

It this section we suppose that all the active forces are conservative. In view of the non-singularity of Hertz matrix $\frac{\partial^{2} T}{\partial q^{\prime} \partial q^{\prime}}$, we can introduce the Legendre's transformation : 


$$
\begin{aligned}
& p_{s}=\frac{\partial}{\partial q_{s}^{\prime}} L\left(q, q^{\prime}, t\right)(s=1, \cdots, n), \\
& H(q, p, t)=\sum_{s=1}^{n} p_{s} q_{s}^{\prime}-L\left(q, q^{\prime}, t\right) .
\end{aligned}
$$

These two equations give

$$
q_{s}^{\prime}=q_{s}^{\prime}(q, p, t)=\frac{\partial H}{\partial p_{s}}(s=1, \cdots, n),
$$

and so $(19),(22),(16),(17)$ and (21) become

$$
\begin{gathered}
\int_{t_{0}}^{t_{1}} \delta\left(\sum_{s=1}^{n} p_{s} \dot{q}_{s}-H\right) \mathrm{d} t-\left.\sum_{s=1}^{n} p_{s} \delta q_{s}\right|_{t_{0}} ^{t_{1}}=0, \\
\int_{t_{0}}^{t_{1}} \delta\left(\sum_{s=1}^{n} p_{s} \dot{q}_{s}-H\right) \mathrm{d} t=0, \\
u_{\beta}(q, p, t)=f_{\beta}\left(q, q^{\prime}, t\right)=0(\beta=1, \cdots, g), \\
\sum_{s=1}^{n} \sum_{k=1}^{n} \frac{\partial u_{\beta}}{\partial p_{k}}\left[\frac{\partial^{2} H}{\partial p^{\partial} p}\right]_{n s}^{-1} \delta q_{s}=0(\beta=1, \cdots, g), \\
\delta n_{s}\left(i_{0} ;=: \delta p_{s}\left(t_{1}\right)=0 i s=1, \cdots, n\right),
\end{gathered}
$$

respectively. Thus we obt:iin the follcwing two statements:

Theorens 5 ! :Hamilton representation of variational principle of the second kind with free end-points). The smooth function $(q(t), p(t))$ defined in time interval $\left(t_{0}, t_{1}\right)$ is an actual motion of Hamilton system under condition (30) (i.e. conditions (2) and (3)), if and only if it satisfies (30) for every moment of time $t \in\left(t_{0}, t_{1}\right)$ and the partial variational equation (28) with all the smooth variations $\delta p$ and all the $\delta q$ satisfying condition (31).

Theorem 6 (Hamilton representation of variational principle of second kind with half-fixed (or fixed) end points). The smooth function $(q(t), p(t))$ defined in time interval $\left(t_{0}, t_{1}\right)$ is an actual motion of Hamilton system under condition (30) (i.e. conditions (2) and (3)), if and only if it satisfies (30) for every moment of time $t \in\left(t_{0}, t_{1}\right)$ and the partial variational equation (29) with all the smooth variations $\delta p$ (or satisfying condition (32)) and all the $\delta q$ satisfying conditions (31) and (20).

Although all the variational principles given above are related to Newton system, it is easy to prove that Lagrange representation and Hamilton representation of the variational principles with the words related to Newton system deleted still exist for Lagrange systems and Hamilton systems themselves respectively. Besides, when the non-holonomic constraint conditions do not exist, our variational principles will reduce to those for holonomic mechanics, with the well-known Livens Theorem ${ }^{[8]}$ and "Modified Hamilton Principles" $[9,10]$ being two examples .

\section{Leading to integral variational principles of first kind and intermediate kinds}

Since the actural motion condition (1) is a deduction of any principle of the second kind, we will get a new principle equivalent to the original one if condition (1) is put on the trial actual motion in the original principle. Then in the new principle, combining condition (1) of the trial actual motion with partial variational equation (22) we obtain a new partial variational equation

$$
\int_{t_{0}}^{t_{1}}\left[\delta L+\sum_{s=1}^{n}\left(\frac{\partial L}{\partial q_{s}^{\prime}}\left(\delta \dot{q}_{s}-\delta q_{s}^{\prime}\right)+Q_{s}^{\prime} \delta q_{s}\right)\right]_{q^{\prime}=q} \mathrm{~d} t=0 .
$$


Notice that the subscript $q^{\prime}=\dot{q}$ is only put on trial actual motion, but not on any comparative motion, so $\delta q^{\prime}{ }_{s}$ is still independent variation (of course the end-point condition must be satisfied). Therefore the new principle still belongs to those of second kind. However, expanding $\delta L$ in (33) shows that the terms containing $\delta q_{s}^{\prime}$ are canceled out so that all selection methods of $\delta q_{s}^{\prime}$ are equivalent. Thus in the new variational principle, imposing any linear relation (called general relation of $\delta q^{\prime}-\delta q$ )

$$
\delta q_{s}^{\prime}=A_{s}(\delta q)(s \in\{1, \cdots, n\})
$$

(here, $A_{s}$ may depend on trial actual motiom $q, q^{\prime}$ and $t$ ) on $\delta q^{\prime}$ is equivalent to permitting $\delta q^{\prime}$ to be independent. But the new variational principle with condition (34) just belongs to those of the first kind (for the number $M_{s}$ of $s$ is $n$ ) or intermediate kinds (for $0<M_{s}<n$ ). Therefore we have

Theorem 7 (Lagrange representation for general form of variational principle of first and intermediate kinds with half-fixed (or fixed) end points). The sufficiently smooth function $\left(g(t), q^{\prime}(t)\right)$ defined on time interval $\left[t_{0}, t_{1}\right]$ is an actual motion of Lagrange system with constraint condition (16), if and only if it satisfies eq. (16) for every moment of time $t \in\left[t_{0}, t_{1}\right]$ and the partial variational equation (33) with $\left(\delta q, \delta q^{\prime}\right)$ satisfying condition (34). Aptell Cheiaev conditions (17) and end-point conditions (20) (or plus equation (21)).

With each specified form of $A_{s}$, a paricuiar vanational principle is obtained. It is worth noticing that as shown by the above deduction, the form of $A_{s}$ can be independent of constraint condition (16). Besides, when the non-thclonomic constraint conditions do not exist, the general form of the variational principles of the first kind and intermediate kinds will reduce to those for holonomic mechanics.

Take some examples as follows: (i) Let eq. (34) take the form $\delta q^{\prime}=0$. Then eq. (33) takes the partial variational form $\int_{t_{0}}^{t_{1}}\left[\delta L\left(q, q^{\prime}, t\right)+\sum_{s=1}^{n}\left(\frac{\partial L}{\partial q_{s}^{\prime}} \delta \dot{q}_{s}+Q^{\prime}{ }_{s} \delta q_{s}\right)\right]_{q^{\prime}=q} \mathrm{~d} t=0$, whose form has not appeared in literature. (ii) Under the condition that there are no non-conservative active forces, let eq. (34) take the form of $\delta q^{\prime}=\frac{\mathrm{d}}{\mathrm{d} t} \delta q$. Then eq. (33) takes Hölder partial variational form ${ }^{[1-4]} \int_{t_{0}}^{t_{1}}[\delta L(q$, $\left.\left.q^{\prime}, t\right)\right]_{q^{\prime}=q} \mathrm{~d} t=0$. (iii) Under the condition that there are no non-conservative active forces, let (34) take Susrov's form. Then (33) takes Susrov's partial variational form ${ }^{[1-4]}$. (iv) Let (34) take the form $\delta q^{\prime}{ }_{s}=\delta \dot{q}_{s}+\left(Q^{\prime}{ }_{s} / \frac{\partial L}{\partial q_{s}^{\prime}}\right) \delta q_{s} \quad(s=1, \cdots, n)$. Then (33) becomes $\int_{t_{0}}^{t_{1}}\left[\delta L\left(q, q^{\prime}, t\right)\right]_{q^{\prime}=q} \mathrm{~d} t=0$.

\section{References}

1 Rumyantsev, V. V., Proc. IUTAM-ISIMM Symp. on Modern Developments in Analytical Mechanics, Torino, 1982, 697716

2 Fengxiang, M., Foundation of Holonomic Mechanics (in Chinese), Beijing: Beijing Tech. Univ. Press, $1985,63$.

3 Bin, C., Analytical Dynamics (in Chinese), Beijing: Beijing Univ. Press, 1987, 345-400; 64-67.

4 Arnold, V. L., Dynamical System, Vol. 3, Berlin: Springer, 1988, 9-20.

5 Bin, C., On an argument on non-holonomic mechanics, Acta Mech. Sinica, 1991, 23(3): 379.

6 Liang Lifu, Shi Zhifei, On the selected regions of general displacements in non-holonomic systems, Acta Mech. Solida Sinica (in Chinese), 1993, 14(3): 189 .

7 Cushing, J. T., Applied Analytical Mathematics for Physical Scientsts, New York: John Wiley \& Sons, $1975,237$.

8 Pars, L. A., A Treatise on Analytical Dynamics, London: Heinemann, 1965, 530-531.

9 Desloge, E. A., Classical Mechanics, Vol. 2, New York: John Wiley \& Sons, 1982, 483-852.

10 Vujanovic, B., A variational principle for non-conservative dynamical systems, .Z Angew Math. Mech., $1975,55: 321$. 